\title{
Inflammatory Events in Patients with Sagittal Imbalance through Platelet-Associated Inflammation
}

\author{
Arsal ACARBAS \\ Mugla Sitki Kocman University, Faculty of Medicine, Department of Neurosurgery, Mugla, Turkey \\ Corresponding author: Arsal ACARBAS arsal5@hotmail.com
}

\section{ABSTRACT}

AIM: To determine whether platelet-associated inflammation parameters (PAIP) could be useful for evaluating inflammation in sagittal imbalance $(\mathrm{SI})$ by investigating its relationship with pelvic tilt (PT), sacral slope (SS), pelvic incidence (PI), lumbar lordosis (LL) and thoracic kyphosis (TK).

MATERIAL and METHODS: The study included 57 patients (19 males, 38 females) who were diagnosed with SI and 44 healthy control subjects. Spinal sagittal balance was evaluated using digitalized-standing lumbar lateral radiographs of the patients. All parameters including SS, PI, LL and PT were evaluated through from the lateral radiographs.

RESULTS: In the analysis, Mean Platelet Volume (MPV) $(10.6 \pm 0.9 ; \mathrm{p}<0.0001)$, Platelet Distribution Width (PDW) (12.7 \pm 2.2 ; $\mathrm{p}<0.001)$ and Platelet Distribution Width $(\mathrm{RDW})(41.9 \pm 4.4 ; \mathrm{p}<0.0001)$ were observed to be much higher in the SI than in the control. The neutrophil-lymphocyte ratio (NLR) was higher in the SI group $(p=0.033)$, and no difference was determined in respect of the platelet-lymphocyte ratio (PLR) ( $p>0.05)$. The PT had a positive correlation with hospital length of stay in SI patients $(r=0.655$; $p=0.008)$. MPV showed a strong positive correlation with comorbidity $(r=0.715 ; p=0.001)$. A positive correlation was determined between NLR and PLR ( $r=0.692 ; p=0.002)$, and between MPV and RDW ( $r=0.788 ; p=0.001)$. No correlation was determined between the PAIP and radiological measurements (PI, PT, SS) that were used for SI.

CONCLUSION: The results suggest that the neurosurgical approach should cover not only the sagittal parameters and their angles but also PAIP showing inflammatory events originating from platelets.

KEYWORDS: Spine, Sagittal imbalance, Platelet, Inflammation

\section{INTRODUCTION}

S agittal imbalance (SI) in the spine includes kyphosis and lordosis in the form of an inconsistent state (1). In a standing person, the center of mass of the body is located in front of the thoracic spine and near the lumbar region (32). In this way, the person can stand upright with support from the ground. If we look at a person in normal sagittal equilibrium, in which these two lines are in harmony, it can be seen that the projection of the head is inside the pelvis (7). In pathological conditions in which sagittal balance is impaired, the organism tries to maintain the sagittal balance by numbing the line of tension and the weight line with each other (16). That results in the development of restricted mobility with a low quality of life, which is accepted as the most critical reason for spinal surgery (29). With the deterioration of a systemic or functional unit, compensation mechanisms develop to provide the sagittal balance against changes in the spine $(1,33)$. When the spine begins to change shape, changes also occur in the pelvis in an effort to keep sagittal balance strong. As neurosurgeons, we evaluate these changes in the spine using spinopelvic measurements such as lumber lordosis (LL), pelvic incidence $(\mathrm{PI})$, sacral slope (SS), pelvic tilt (PT), and thoracic kyphosis (TK) (9).

Considering the occurrence of the SI, degenerative disc disease, lumbar disc hernia, degenerative and isthmic spondylolisthesis and similar pathologies, changes in the spine and 
pelvis as a result of the complementary mechanisms cause inflammation around the resistant pain and deterioration in quality of life $(9,15)$. All these events caused by inflammatory conditions have been the major reasons of back pain affecting people (19). Recent studies have pointed out that increased inflammatory conditions strongly affect the efficacy of surgical intervention in the affected spinal areas and the recovery period after surgery (21). Evaluation of the inflammatory condition may allow important predictions of the effectiveness of the surgical procedure and postoperative recovery (28). Recent studies have shown a significant relationship between platelets and inflammatory events in several diseases $(2,17,23,30)$. Therefore, it can be considered that novel platelet-associated inflammation parameters (PAIP) such as Platelet Distribution Width (PDW), Mean Platelet Volume (MPV), Platelet-to-Lymphocyte Ratio (PLR) and Neutrophil-to-Lymphocyte Ratio (NLR) could provide information about inflammatory events.

The aim of the current study was to determine whether PAIP could be useful in the evaluation of inflammation in SI by investigating its relationship with SS, PI, PT, LL and TK.

\section{MATERIAL and METHODS}

\section{Study Design}

Adult SI patients were examined by a single surgeon in Mugla Sitki Kocman University, Faculty of Medicine Hospital, between June 2015 and February 2019 in this single-center retrospective cohort study. A total of 57 patients (male/ female: 19/38) who were diagnosed with SI and needed a posterior-approach spinal surgery of L4 and L5 including posterior instrumentation, decompression, and lateral fusion with/without lumbar-interbody fusion were enrolled in this study. The healthy individuals were selected from laboratory and examination records obtained from the hospital patient information system. All the patients and controls were aged higher than 18 years and all provided written-informed consent for participation in the study. Any procedures in the current study were in accordance with the Decleration of Helsinki. The Human Experiment and Ethical Committee of Mugla Sitki Kocman University Medical Center approved all the procedures (Decision date and number: 02.May.2019 and 08/ IX).

\section{Radiological Evaluation}

Lateral radiographs were analysed in all patients with SI. Patient records were obtained from the hospital electronic database. The presentation of lower back pain with intermittent claudication or sciatica and radiographic image showing SI findings was accepted as the reference for the diagnosis. Spinal sagittal balance was evaluated using digitalizedstanding lumbar lateral radiographic images of the patients (Figure 1, 2). The standing position was used to obtain the preoperative films, radioscopy to see the placement of the pedicle screws was used for intraoperative films. All patients used a standard pad in the position of prone decubitus to provide $40^{\circ}$-hip flexion and $30^{\circ}$-knee flexion, both measured with an angle ruler.
All parameters for SI were measured and evaluated through from the radiographs. The PI is morphological/anatomical measurement independent of the pelvis spatial orientation. PI measurement that we used was as followings: we provided a line on the S1 superior end plate; found the middle point for this and draw a vertical line downwards; pointed a line of the head of the central sacrum line from the head of the femoral headline. Calculating the angle in this line provided us PI. Angulation of the inferior angle of T12 with the superior end plate of S1 provided us the SI. The PT was obtained by the angle between the two lines that involves one line found by drawing a line from the midpoint of the sacral end plate running perpendicular down to the bottom of the X-ray; and by the second line by drawing a line from the centre of the femoral heads to the center of the sacrum. We found "PI" adding "PT" to "SS" as another way. The SS was found by a line draw along the superior sacral-end-plate in addition to another line draw from the anterior superior-edge parallel to the bottom of the X-ray.

\section{Laboratory Analysis for PAIP}

Venous blood samples were taken within 24 hours preoperatively for complete blood count (CBC) analysis. Immediate analysis was applied to determine leukocyte count, neutrophil count, platelet count, MPV, PDW and RDW. The ratio of PAIP was calculated from the neutrophil, platelet and lymphocyte count values. Haematological parameters were measured using an automated haematology analyser (Coulter LH 780 Haematology Analyser, Beckman Coulter Inc, CA, USA). The upper limits of the reference intervals were as follows: Leuko-

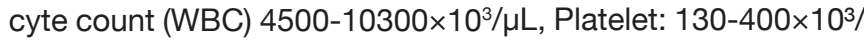

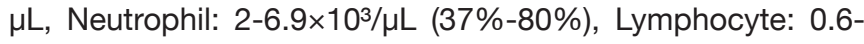
$3.4 \times 10^{3} / \mu \mathrm{L}$ (10\%-50\%), MPV: $7.2-11 \mathrm{fL}, \mathrm{RDW}: 11 \%-16 \%$, and PDW: $10 \%-18 \%$. The haematological parameters were assayed in the ethylene diamine tetraacetic acid treated (EDTA) blood samples within 1 hour of vein puncture.

\section{Statistical Analyze}

Data of the present study were analysed statistically using SPSS Windows version 20 software (SPSS Inc., Chicago, IL, USA). All data were expressed as mean and standard deviation (mean $\pm \mathrm{SD}$ ) values. Distribution of data was assessed using the Shapiro-Wilk test. The Chi-square test was applied to comparisons of groups of categorical variables. For independent samples, the t-test was applied to continuous variables. The Pearson or Spearman test was performed for the correlation analysis according to the Shapiro-Wilk test results. A value of $p<0.05$ was considered statistically significant. All outcomes were evaluated within a 95\% Confidence-Interval (Cl).

\section{RESULTS}

The mean age of SI patients was $54.2 \pm 24$ years (range: $22-78$ years), and of the control group, $49.8 \pm 23$ years (range: 29 71 years) ( $p>0.05$ ). No significant difference was determined between the groups in terms of demographics including age and BMI ( $p>0.05)$. The LL evaluation was defined as 29 normal, 22 hypolordosis, 15 hyperlordosis, and 1 kyphosis. 
Measurement of SS and PT were different within the groups, as seen in Table I.

In the routine biochemistry comparisons between the groups, all parameters were similar except Erythrocyte Sedimentation Rate (ESR) $(24 \pm 19 ; \mathrm{p}<0.0001)$ and C-reaktive protein $(\mathrm{CRP})$ $(7 \pm 7.2 ; p<0.001)$, which were naturally found to be higher in SI patients. No significant differences were observed between the groups in respect of the hormone analyses including $25-\mathrm{OH}$ vitamin $\mathrm{D}$, thyroid stimulating hormone (TSH) and Parathyroid Hormone (PTH) (Table II). In haematological findings, leukocyte, neutrophil, lymphocyte, platelet, red blood cell (RBC) and haemoglobin were within normal ranges. In the PAIP analyses, MPV (10.6 $\pm 0.9 ; \mathrm{p}<0.0001)$, PDW $(12.7 \pm 2.2$; $\mathrm{p}<0.001)$ and $\operatorname{RDW}(41.9 \pm 4.4 ; \mathrm{p}<0.0001)$ were determined to be much higher in the SI group than in the control group. NLR was significantly higher in the SI group $(p=0.033)$, and no difference was determined in respect of PLR ( $p>0.05)$.

A positive correlation was determined between $\mathrm{PT}$ and length of hospital stay in SI patients $(r=0.655 ; p=0.008)$. MPV showed a strong positive correlation with comorbidity $(r=0.715$;
Table I: Demographic Data of the Groups

\begin{tabular}{|c|c|c|c|c|}
\hline \multicolumn{2}{|l|}{ Variables } & Control & Patient & $\mathbf{p}$ \\
\hline \multicolumn{2}{|l|}{ Age (years) } & $49.8 \pm 23$ & $53.2 \pm 24$ & 0.511 \\
\hline \multirow{2}{*}{ Gender (m/f) } & Male & 25 & 19 & \multirow{2}{*}{0.023} \\
\hline & Female & 19 & 38 & \\
\hline \multicolumn{2}{|l|}{ BMI $\left(\mathrm{kg} / \mathrm{m}^{2}\right)$} & $25 \pm 3$ & $26 \pm 3$ & 0.162 \\
\hline \multicolumn{2}{|l|}{ Comorbidity } & - & $1 \pm 1$ & \\
\hline \multicolumn{2}{|c|}{$\begin{array}{l}\text { Length of Hospital Stay } \\
\text { (days) }\end{array}$} & - & $8 \pm 3$ & \\
\hline \multicolumn{2}{|c|}{ Follow-up Period (months) } & - & $2 \pm 2$ & \\
\hline \multirow{4}{*}{$\begin{array}{l}\text { Lumbar } \\
\text { Lordosis }\end{array}$} & Normal & 44 & 29 & \\
\hline & Hypo & 0 & 12 & \\
\hline & Hyper & 0 & 15 & \\
\hline & Kyphosis & 0 & 1 & \\
\hline \multicolumn{2}{|c|}{ Pelvic incidence (PI) } & $54.7 \pm 15.1$ & $53.8 \pm 13.2$ & 0.746 \\
\hline \multicolumn{2}{|c|}{ Sacral slope (SS) } & $40.1 \pm 12.7$ & $30.5 \pm 11.8$ & 0.0001 \\
\hline \multicolumn{2}{|l|}{ Pelvic tilt (PT) } & $18.1 \pm 13.9$ & $23.7 \pm 12.7$ & 0.036 \\
\hline
\end{tabular}
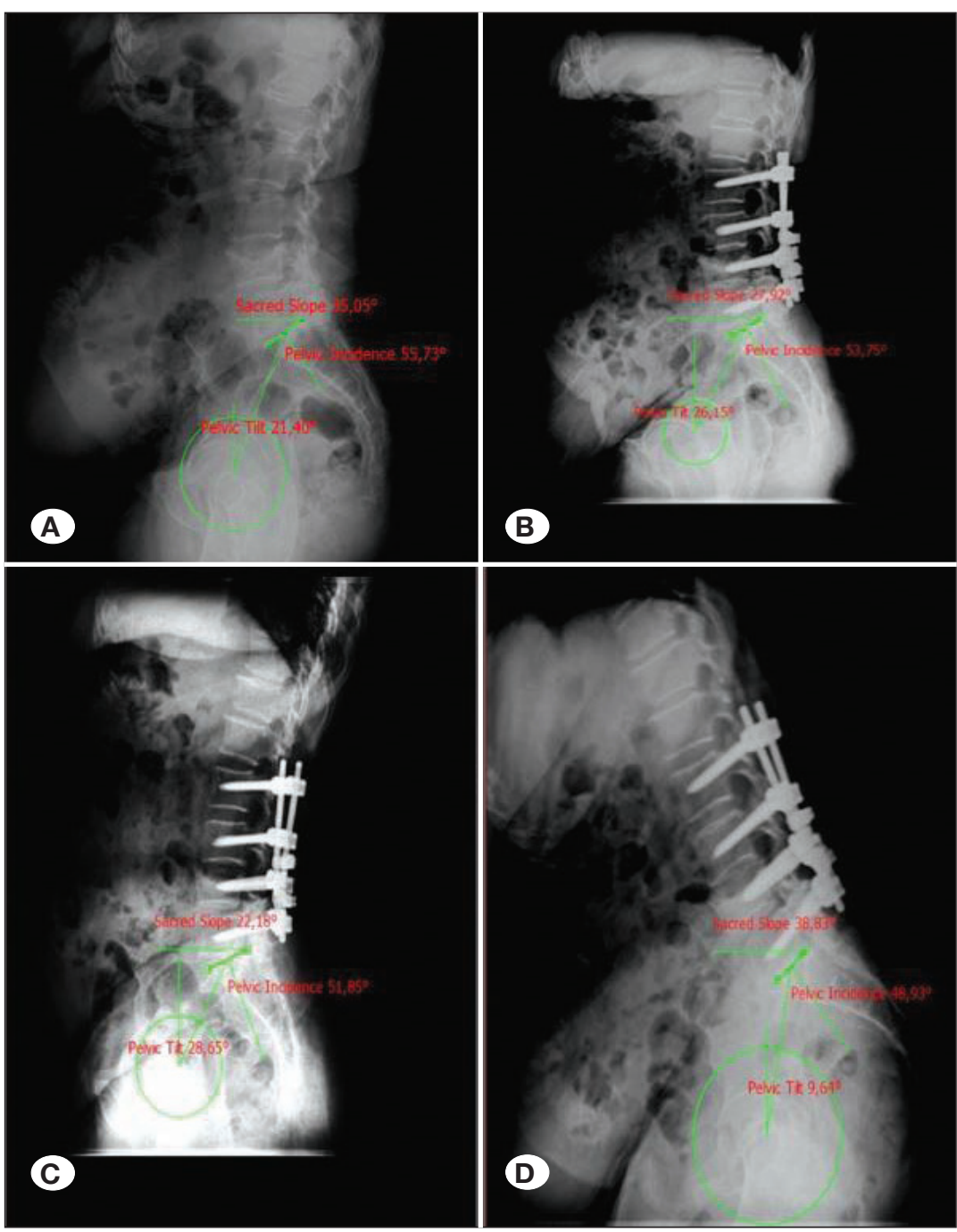

Figure 1: A) Lateral radiograph of a preoperative patient with lumbar spinal pathology. B-D) Postoperative radiological sagittal balance assessments after posterior segmental stabilization between L2 and S1 levels. 


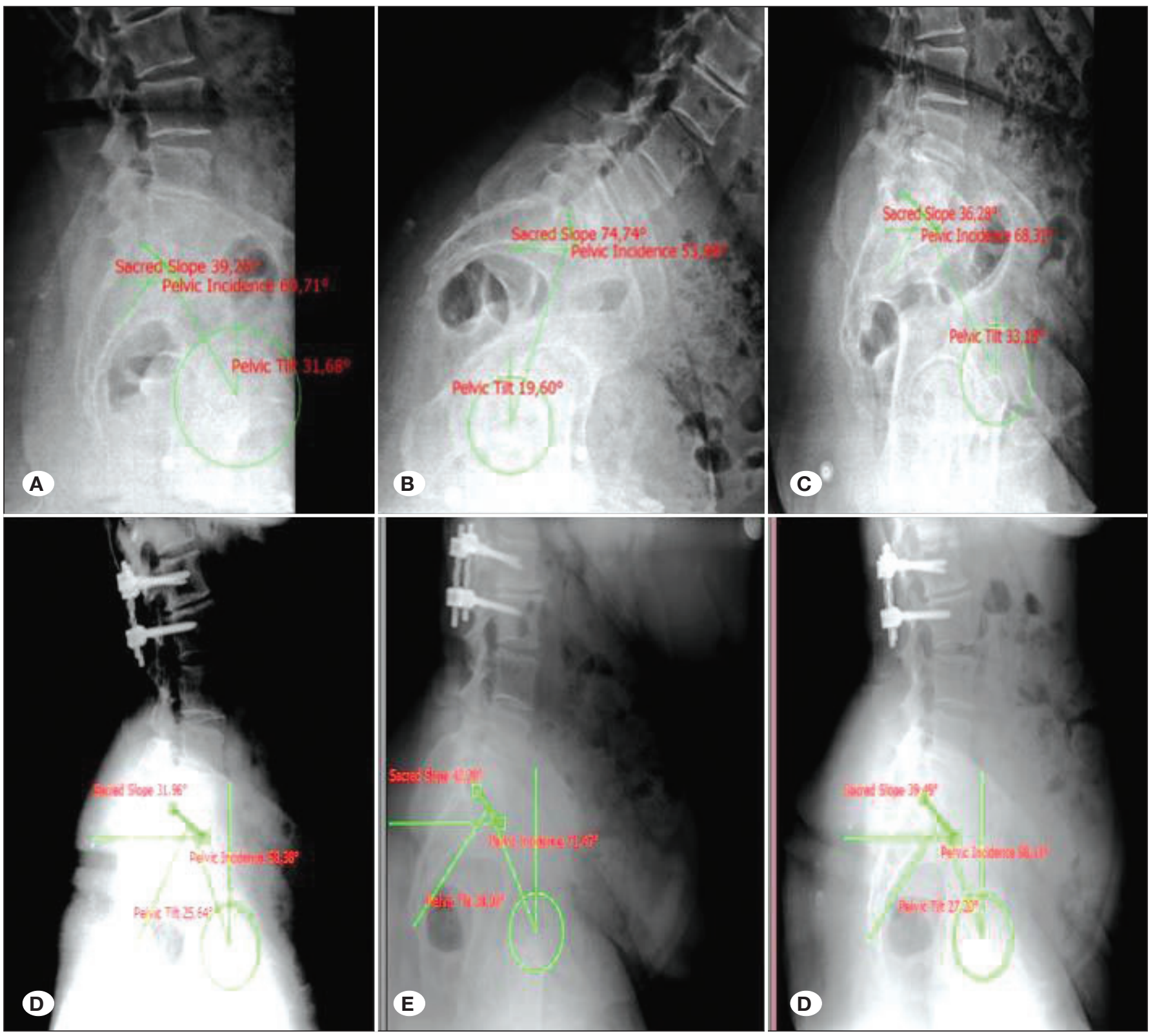

Figure 2: Lateral lumbar spinal radiograps of a patient in neutral (A), flexion (B), and extension (C) positions before surgery. Neutral (D), flexion (E) and extension (F) radiographs (including measurements) of a patient who underwent surgery for lumbar (L) 1-2 extruded disc herniation. Discectomy and dynamic posterior stabilization was performed.

$\mathrm{p}=0.001)$. A positive correlation was determined between NLR and PLR ( $r=0.692 ; p=0.002)$, and between MPV and RDW $(r=0.788 ; p=0.001)$. No correlation was determined between PAIP and the radiological measurements (PI, PT, SS) used for SI.

\section{DISCUSSION}

Surgical restoration of global alignment and minimization of complications through several options can successfully improve the pain and function of sagittal deformity. This restoration cannot be considered without evaluating the inflammatory conditions. The objective of this retrospective cohort study was to determine which PAIP could contribute to understanding inflammatory events in the SI, which has important implications for neurosurgeons. The results of the study suggested that the surgical approach should cover not only the sagittal parameters and angles but also the PAIP showing inflammatory events originating from platelets.

Neurosurgeons must keep in mind that spinal surgeons should carefully consider the spinopelvic parameters in spinal pathologies prior to surgical intervention and perform surgical procedures to provide postoperative balance of spine (4). 
Table II: Laboratory Results of the Groups

\begin{tabular}{|c|c|c|c|}
\hline Variables & Control & Patients & $\mathbf{p}$ \\
\hline Glucose (mg/dL) & $95 \pm 8$ & $97 \pm 10$ & 0.221 \\
\hline Uric Acid (mg/dL) & $4.7 \pm 1.2$ & $4.5 \pm 1.3$ & 0.465 \\
\hline Albumin (g/dL) & $4.5 \pm 0.2$ & $4.5 \pm 0.4$ & 0.344 \\
\hline BUN (mg/dL) & $34.7 \pm 15.2$ & $32.9 \pm 13.8$ & 0.525 \\
\hline Creatinine (mg / L) & $0.7 \pm 0.2$ & $0.8 \pm 0.2$ & 0.209 \\
\hline AST (U/L) & $20 \pm 9$ & $20 \pm 9$ & 0.992 \\
\hline ALT (U/L) & $21 \pm 14$ & $22 \pm 13$ & 0.798 \\
\hline $\mathrm{ESR}(\mathrm{mm} / \mathrm{h})$ & $3 \pm 1$ & $24 \pm 19$ & 0.0001 \\
\hline $\mathrm{CRP}(\mathrm{mg} / \mathrm{dL})$ & $2.5 \pm 1.3$ & $7 \pm 7.2$ & 0.001 \\
\hline Fibrinogen (mg/dL) & $269 \pm 51$ & $277 \pm 71$ & 0.401 \\
\hline Calcium (mg/dL) & $9.1 \pm 0.5$ & $9.3 \pm 0.5$ & 0.069 \\
\hline Magnesium (mg/dL) & $1.9 \pm 0.1$ & $1.9 \pm 0.1$ & 0.345 \\
\hline \multicolumn{4}{|l|}{ ELISA Findings } \\
\hline 25-OH-D (pg/mL) & $19.7 \pm 5.6$ & $22 \pm 7.4$ & 0.094 \\
\hline TSH (ng/ml) & $2.2 \pm 0.95$ & $2.1 \pm 0.88$ & 0.235 \\
\hline Free $\mathrm{T}_{3}(\mathrm{pg} / \mathrm{ml})$ & $1.5 \pm 0.38$ & $1.4 \pm 0.55$ & 0.408 \\
\hline Free $\mathrm{T}_{4}(\mathrm{pg} / \mathrm{ml})$ & $3.1 \pm 0.78$ & $3 \pm 0.51$ & 0.178 \\
\hline PTH (ng/ml) & $29.2 \pm 6.25$ & $31 \pm 4.41$ & 0.589 \\
\hline \multicolumn{4}{|c|}{ Haematological Findings } \\
\hline Leukocyte (x109/L) & $6.2 \pm 1.6$ & $6.3 \pm 1.6$ & 0.645 \\
\hline Neutrophyl (x109/L) & $3.9 \pm 1.2$ & $6.5 \pm 8$ & 0.095 \\
\hline Lymphocyte (x109/L) & $1.7 \pm 0.7$ & $2 \pm 0.8$ & 0.045 \\
\hline Platelet (x10\%/L) & $244 \pm 64$ & $293 \pm 104$ & 0.086 \\
\hline Heamoglobin (g/dl) & $13.3 \pm 1.1$ & $13.2 \pm 1.1$ & 0.574 \\
\hline Hematocrite (\%) & $38.2 \pm 2.8$ & $37.9 \pm 2.7$ & 0.672 \\
\hline $\operatorname{RBC}(\times 10 \% / L)$ & $4.4 \pm 0.4$ & $4.4 \pm 0.4$ & 0.893 \\
\hline $\operatorname{MCV}(f L)$ & $86 \pm 6$ & $86 \pm 7$ & 0.878 \\
\hline $\mathrm{MCH}(\mathrm{pg})$ & $98.3 \pm 453$ & $82.6 \pm 398$ & 0.876 \\
\hline $\mathrm{MCHC}(\mathrm{g} / \mathrm{dL})$ & $34.9 \pm 0.7$ & $34.8 \pm 0.6$ & 0.853 \\
\hline N/L Ratio (NLR) & $2.5 \pm 1.1$ & $4.2 \pm 5.7$ & 0.033 \\
\hline P/L Ratio (PLR) & $158 \pm 74.4$ & $162.3 \pm 74$ & 0.774 \\
\hline MPV (fL) & $8.5 \pm 0.8$ & $10.6 \pm 0.9$ & 0.0001 \\
\hline PDW (\%) & $15.2 \pm 2.2$ & $12.7 \pm 2.2$ & 0.001 \\
\hline RDW (\%) & $14.5 \pm 1.5$ & $41.9 \pm 4.4$ & 0.0001 \\
\hline
\end{tabular}

If the $\mathrm{LL}$ and spinopelvic adaptation required to restore the balance cannot be achieved after the surgical interventions, the expected positive response from the surgical treatment will not be attained due to the continued SI (15). Kocyigit et al reported that measurements taken from radiographs such as PI, SS, PT, and LL, have a critical role in providing sufficient information about SI (14). For example, ankylosing spondylitis is a chronic inflammatory rheumatic disease characterized by inflammation of the vertebrae, which progresses to bone fusion of the spinal column (33). Sagittal spinal balance can be influenced from the onset of the disease and can result in the development of a rigid, non-flexible spinal column and thoracolumbar kyphosis (24). Therefore, the aim of this study was to investigate if these measurements can provide information not only for conditions of SI but also for events with an inflammatory process.

The synovial tissue and cartilage of the facets contain inflammation related cytokines, which may be in touch with a nerve root, and as reported by Cohen and Raja, with the occurrence of pathological changes in nerve tissue, behavioural deficits may be seen (3). All the reports show that radicular symptoms can be induced by biochemical factors generated by facet degeneration affecting to nerve roots. Increased expression of interleukins in the facets supports the outcome that biochemical inflammatory agents may be the major reason (20). It is clear that there are inflammatory events in diseases that progress to conditions of SI. Tsang and Chung stated that CRP was the most useful tool in screening for axial-joint inflammation (27), and this test is still accepted as useful despite its low sensitivity and specificity. This also led to a search for novel useful markers similar to CRP. Recent studies have shown platelets to be the most important potential inflammatory candidates and changing values could be useful to demonstrate events of SI $(2,30)$. Therefore, platelet-related parameters were analysed in this study in addition to CPR and ESR. As previously reported, the ESR and CRP values in the current study patients were found to be reased as expected in SI $(3,27)$. However, it is clear that additional parameters are necessary for further evaluations of inflammation in SI.

The benefit of using PAIP has been shown in the evaluation of systemic inflammation in various health problems such as diabetes mellitus, ulcerative colitis, cirrhosis and cancer [18-24]. In recently, increased level of PLR was strongly associated with activity and severity of psoriasis and RA, as well as psoriatic arthritis $(8,12,31)$. Apart from rheumatology disorders, higher PLR, as a ratio of inflammation, behaves to be associated with lessened survival in gastric and pancreatic malignities (25). Similarly, levels of MPV were investigated widely in the recent decade. Increased MPV was on the other hand mainly found in states of low grade inflammation such as: cellulitis, idiopathic subjective tinnitus, symptom/attackfree periods of familial Mediterranean fever patients and irritable bowel syndrome, obstructive sleep apnea syndrome $(5,6,11,22)$.

In a few recent studies, the NLR value has been determined to be higher in RA patients compared to control subjects, and to be associated with disease activity (25-27). Kantola 
et al. reported that there was a positive correlation between increased NLR in neoplastic disorders and certain cytokines (10), some of which are present in the pathogenesis of RA and AS $(18,26)$. According to Kisacik et al., MPV values in RA could provide additional information about inflammatory events (13). While a positive correlation between MPV and ESR was reported in a study by Sag et al., no correlation with CRP was found (23). In the current study, MPV and PDW showed a significant increase in the SI patients although NLR showed only slight significance in the SI patients compared to the control group. In contrast, PLR, which is accepted as a significant inflammatory indicator, had no significance when the SI group was compared with the control group. Unlike other relevant studies, Sag et al. found no relationship between CRP and PAIP such as NLR, PLR, MPV, PDW (23). Similar to that study, no significant correlations were determined between CRP and PAIP in the current study patient group. Although the SS and PT values varied significantly in the SI patients, the value of PI was not different from that of the control group. When the correlations were considered, the PT value had a positive correlation with length of hospital stay in the SI patients. MPV demonstrated a strong positive correlation with comorbidity. In contrast, there was no correlation between PAIP and the radiological measurements (PI, PT, SS) used for SI.

This study had limitations in terms of design and capacity. As the study was retrospective and cross-sectional, it was not possible to analyse inflammatory cytokines such as IL-1, TNF-a, IL-10 and rheumatology-related biomarkers such as ANA, ASA, RF in these patients. A second limitation was the small number of patients for a retrospective analysis and the relationship of PAIP with pain scores could not be evaluated, as it was not a prospective analysis. In addition, any difference between the deformity patients regarding inflammatory parameters may change the outcome scores. We were unable to indicate if any relation between the surgical results and inflammatory parameters in the SI patients. The diagnostic history could not be fully determined from the patient database for all the SI patients and thus the comprehensive diagnosis was accepted as SI.

\section{CONCLUSION}

The results of this study suggest that the neurosurgical approach should cover not only the sagittal parameters and their angles but also PAIP showing inflammatory events originating from platelets. There is still much to be learned about the interactions and the influence of the many components that regulate the inflammatory process in SI. It can be considered that improvements in the knowledge and understanding of platelet-related inflammation and its effects on spinal surgery could improve future diagnostic and therapeutic approaches.

\section{REFERENCES}

1. Chun SW, Lim CY, Kim K, Hwang J, Chung SG: The relationships between low back pain and lumbar lordosis: A systematic review and meta-analysis. Spine Journal 17: 11801191, 2017
2. Cintesun E, Incesu Cintesun FN, Ezveci H, Akyurek F, Celik C: Systemic inflammatory response markers in preeclampsia. $\mathrm{J}$ Lab Physicians 10:316-319, 2018

3. Cohen SP, Raja SN: Pathogenesis, diagnosis, and treatment of lumbar zygapophysial (facet) joint pain. Anesthesiology 106:591-614, 2007

4. Coskun Benlidayi I, Basaran S: Comparative study of lumbosacral alignment in elderly versus young adults: Data on patients with low back pain. Aging Clinical and Experimental Research 27:297-302, 2014

5. Delgado-Garcia G, Galarza-Delgado DA, Colunga-Pedraza I, Borjas-Almaguer OD, Mandujano-Cruz I, Benavides-Salgado D, Martinez-Granados RJ, Atilano-Diaz A: Mean platelet volume is decreased in adults with active lupus disease. Revista Brasileira de Reumatologia 56:504-508, 2016

6. Erturk A, Cure E, Cure MC, Parlak E, Kurt A, Ogullar S: The association between serum YKL-40 levels, mean platelet volume, and c-reactive protein in patients with cellulitis. Indian journal of medical microbiology 33 Suppl:61-66, 2015

7. Evcik D, Yucel A: Lumbar lordosis in acute and chronic low back pain patients. Rheumatology International 23:163-165, 2003

8. Fu H, Qin B, Hu Z, Ma N, Yang M, Wei T, Tang Q, Huang $Y$, Huang F, Liang Y, Yang Z, Zhong R: Neutrophil- and plateletto-lymphocyte ratios are correlated with disease activity in rheumatoid arthritis. Clinical Laboratory 61:269-273, 2015

9. Gottfried ON, Daubs MD, Patel AA, Dailey AT, Brodke DS: Spinopelvic parameters in postfusion flatback deformity patients. Spine Journal 9:639-647, 2009

10. Kantola T, Klintrup K, Väyrynen JP, Vornanen J, Bloigu R, Karhu T, Herzig KH, Näpänkangas J, Mäkelä J, Karttunen TJ, Tuomisto A, Mäkinen MJ: Stage-dependent alterations of the serum cytokine pattern in colorectal carcinoma. British Journal of Cancer 107:1729-1736, 2012

11. Kapsoritakis AN, Koukourakis MI, Sfiridaki A, Potamianos SP, Kosmadaki MG, Koutroubakis IE, Kouroumalis EA: Mean platelet volume: A useful marker of inflammatory bowel disease activity. American Journal of Gastroenterology 96: 776-781, 2001

12. Kim DS, Shin D, Lee MS, Kim HJ, Kim DY, Kim SM, Lee MG: Assessments of neutrophil to lymphocyte ratio and platelet to lymphocyte ratio in Korean patients with psoriasis vulgaris and psoriatic arthritis. Journal of Dermatology 43:305-310, 2016

13. Kisacik B, Tufan A, Kalyoncu U, Karadag O, Akdogan A, Ozturk MA, Kiraz S, Ertenli I, Calguneri M: Mean platelet volume (MPV) as an inflammatory marker in ankylosing spondylitis and rheumatoid arthritis. Joint Bone Spine 75:291-294, 2008

14. Kocyigit B, Nacitarhan V, Koca T, Berk E: Comparison of sagittal lumbosacral parameters in patients with ankylosing spondylitis and chronic mechanical back pain. Annals of Medical Research 26(2):199-203, 2019

15. Kocyigit BF, Berk E: Comparison of lumbosacral alignment in Geriatric and Non-Geriatric patients suffering low back pain. Pak J Med Sci 34(2):282-287, 2018

16. Lee JS, Suh KT, Kim JI, Goh TS: Analysis of sagittal balance of ankylosing spondylitis using spinopelvic parameters. Journal of Spinal Disorders and Techniques 27:E94-E98, 2014 
17. Luo WJ, Zhang WF: The relationship of blood cell-associated inflammatory indices and diabetic retinopathy: A Metaanalysis and systematic review. Int J Ophthalmol 12:312-323, 2019

18. Maden M, Pamuk GE, Pamuk ON: Development of atherosclerotic cardiovascular mortality in gouty arthritis and rheumatoid arthritis patients: Are they associated with mean platelet volume and neutrophil-lymphocyte ratio? A comparative study. Arch Rheumatol 32:39-45, 2017

19. Maksymowych WP, Morency N, Conner-Spady B, Lambert RG: Suppression of inflammation and effects on new bone formation in ankylosing spondylitis: Evidence for a window of opportunity in disease modification. Annals of the Rheumatic Diseases 72:23-28, 2012

20. Ng L, Chaudhary N, Sell P: The efficacy of corticosteroids in periradicular infiltration for chronic radicular pain. Spine 30 : 857-862, 2005

21. Park JW, Kim MJ, Lee JS, Ha Y-J, Park JK, Kang EH, Lee YJ, Song YW, Lee EY: Impact of tumor necrosis factor inhibitor versus nonsteroidal antiinflammatory drug treatment on radiographic progression in early ankylosing spondylitis: Its relationship to inflammation control during treatment. Arthritis \& Rheumatology 71:82-90, 2018

22. Safak S, Uslu AU, Serdal K, Turker T, Soner S, Lutfi A: Association between mean platelet volume levels and inflammation in SLE patients presented with arthritis. African Health Sciences 14:919-924, 2014

23. Sag S, Sag MS, Tekeoglu I, Kamanli A, Nas K, Acar BA: Relationship of hematologic markers with IL-17 and IL-1 beta in patients with rheumatoid arthritis. J Back Musculoskelet Rehabil 31:703-707, 2018

24. Sawacha Z, Carraro E, Del Din S, Guiotto A, Bonaldo L, Punzi L, Cobelli C, Masiero S: Biomechanical assessment of balance and posture in subjects with ankylosing spondylitis. Journal of NeuroEngineering and Rehabilitation 9:63, 2012
25. Tasoglu I, Sert D, Colak N, Uzun A, Songur M, Ecevit A: Neutrophil-lymphocyte ratio and the platelet-lymphocyte ratio predict the limb survival in critical limb ischemia. Clinical and applied thrombosis/hemostasis. Journal of the International Academy of Clinical and Applied Thrombosis/Hemostasis 20:645-650, 2014

26. Tekeoglu I, Gurol G, Harman H, Karakece E, Ciftci IH: Overlooked hematological markers of disease activity in rheumatoid arthritis. Int J Rheum Dis 19:1078-1082, 2016

27. Tsang HHL, Chung HY: The discriminative values of the bath ankylosing spondylitis disease activity index, ankylosing spondylitis disease activity score, c-reactive protein, and erythrocyte sedimentation rate in spondyloarthritis-related axial arthritis. Journal of Clinical Rheumatology 23:267-272, 2017

28. Ucak M: Incidence and severity of maxillofacial injuries during the Syrian civil war in syrian soldiers and civilians. Journal of Craniofacial Surgery 30:992-995, 2019

29. Ucak M: Myelomeningocele closure by unilateral lumbar artery perforator flap: Experience with thirty-eight patients. Microsurgery 38:752-757, 2018

30. Ulutas KT, Sarici IS, Arpaci A: Comparison of platelet distribution width and CA19-9 in resectable pancreas cancer. Med Arch 72:210-213, 2018

31. Uslu AU, Kucuk A, Sahin A, Ugan Y, Yilmaz R, Gungor T, Bagcaci S, Kucuksen S: Two new inflammatory markers associated with Disease Activity Score-28 in patients with rheumatoid arthritis: Neutrophil-lymphocyte ratio and plateletlymphocyte ratio. International Journal of Rheumatic Diseases 18:731-735, 2015

32. Vaz G, Roussouly P, Berthonnaud E, Dimnet J: Sagittal morphology and equilibrium of pelvis and spine. European Spine Journal 11:80-87, 2001

33. White AA, Panjabi MM, Thomas CL: The clinical biomechanics of kyphotic deformities. Clin Orthop Relat Res 128:8-17, 1977 